\title{
30. THE DISTRIBUTION IN GALACTIC LONGITUDE OF OBSERVABLE PULSARS
}

\author{
G. LYNG $\AA$
}

Lund Observatory, Lund, Sweden

\begin{abstract}
I have studied the galactic longitude distribution of low latitude pulsars on the assumption that pulsars are evenly distributed in the galactic disk but that their observability is severely impaired by spiral features, rich in $\mathrm{H} I \mathrm{l}$ regions, in which the high electron density causes a high dispersion measure for a comparatively short optical path. The assumption of even distribution in the galactic disk seems natural as no correlation between positions of pulsars and spiral tracers is known, and since supernovae in external galaxies are not known to confine to spiral arms.

There are 14 pulsars in the consistently surveyed interval $l^{\mathrm{II}}=250^{\circ}-70^{\circ}, b^{\mathrm{II}}=$ $-5^{\circ}-+5^{\circ}$. In the intervals $l^{\mathrm{II}}=285^{\circ}-295^{\circ}$ and $l^{\mathrm{II}}=305^{\circ}-45^{\circ}$ the observations are made against the background of the Carina arm and the Sagittarius arm, respectively. Intervals $l^{I I}=250^{\circ}-285^{\circ}$ and $l^{I I}=45^{\circ}-70^{\circ}$ are considered to be inter-arm regions. The interval $l^{\mathrm{II}}=295^{\circ}-305^{\circ}$ contains a relatively weak link of $\mathrm{H}_{\mathrm{II}}$ regions, and is for the present discussion also considered as an inter-arm region. With these definitions the pulsar statistics are presented in Table I.
\end{abstract}

TABLE I

Statistics for low latitude pulsars

\begin{tabular}{lccc}
\hline Description of regions & Total interval in $l^{\text {II }}$ & $\begin{array}{l}\text { Number of } \\
\text { pulsars }\end{array}$ & $\begin{array}{l}\text { Mean dispers } \\
\text { measure }\end{array}$ \\
Towards spiral features & $110^{\circ}$ & 4 & $50 \mathrm{pc} \mathrm{cm}^{-3}$ \\
Inter-arm regions & $70^{\circ}$ & 10 & $107 \mathrm{pc} \mathrm{cm}^{-3}$
\end{tabular}

The data of Table I clearly show that, on the whole, pulsars are seen at larger distances in the inter-arm regions than towards spiral features. If it is assumed that the pulsars viewed towards spiral features evenly fill out the volume considered, then a mean electron density $\bar{n}_{\mathrm{e}}=0.04 \mathrm{~cm}^{-3}$ can be derived and this value in turn corresponds to an effective horizon at $3 \mathrm{kpc}$ for the inter-arm directions. This calculation assumes a constant luminosity function.

The mean distance from the apparent galactic plane for the pulsars studied would, if pulsars are distributed symmetrically in relation to the actual galactic plane, imply a position of the sun $40 \mathrm{pc}$ above this plane.

\section{Acknowledgement}

I thank Professor B. Y. Mills for helpful comments and for the communication of unpublished data. 